\title{
Theoretical and experimental investigations of oversized Ka-band surface-wave oscillator based on 2D periodical corrugated structure
}

\author{
N.S. Ginzburg, E.V. Ilyakov, I.S. Kulagin, N.Yu. Peskov, A.S. Sergeev and V.Yu. Zaslavsky \\ Institute of Applied Physics of the Russian Academy of Sciences, Nizhny Novgorod, Russia, peskov@appl.sci-nnov.ru
}

\section{Introduction}

For spatially-extended relativistic electron beams of sheet and tubular geometry the use of a two-dimensional (2D) distributed feedback was proposed for providing spatial coherence of radiation and increase the total radiation power [1]. Such 2D feedback can be realized in planar and co-axial 2D Bragg structures (resonators) having double-periodic corrugations. Experimental studies of FEMs based on novel feedback mechanism are performed in Ka-band (coaxial geometry) at the University of Strathclyde (Glasgow, UK) [2] and in W-band (planar geometry) at the Budker INP RAS (Novosibirsk, Russia) [3] in collaboration with the IAP RAS (N.Novgorod, Russia) and KIT (Karlsruhe, Germany). As a result, narrow-band multi-MW power radiation was obtained.

At present, theoretical and experimental studies of Cherenkov masers with 2D distributed feedback are in progress [4]. Among relativistic masers of such type, surface wave oscillators (SWO) are beneficial due to the large values of the electron-wave coupling impedance. Besides, formation of a surface mode ensures regular field distribution along the coordinate directed perpendicularly to the corrugated surface and, thus, would solve the problem of mode selection over this coordinate. In SWO 2D periodic structure can be exploited both as a slow-wave system and as a high selective Bragg resonator simultaneously [5]. In this case such structure would provide effective mode control over azimuthal coordinate.

Present paper is devoted to theoretical and experimental studies of oversized SWO with 2D-periodical corrugated structure of cylindrical geometry. Simulations of nonlinear dynamics of the oscillator were performed in the frame of quasi-optical approach and using 3D PIC code CST Studio Suite. Experimental studies were performed at IAP RAS based on the thermionic accelerator "Saturn".

\section{Nonlinear dynamics of SWO with $2 \mathrm{D}$ periodical grating in the frame of quasi-optical approach}

To study nonlinear dynamics of SWO with $2 \mathrm{D}$ periodical grating based on oversized cylindrical waveguide we assume that a hollow rectilinear electron beam moves with longitudinal velocity $V_{0}=\beta_{0} c$ along the $2 \mathrm{D}$ grating (Fig. 1)

$$
r=\frac{r_{1}}{4}\left[\cos \left(\bar{M} \varphi-\bar{h}_{z} z\right)+\cos \left(\bar{M} \varphi+\bar{h}_{z} z\right)\right],
$$

which represents a superposition of two helical corrugations with opposite rotations having period $d_{\mathrm{z}}$ and number of turns $\bar{M}$ (variations of the corrugation over azimuth), $\bar{h}_{z}=2 \pi / d_{z}, r_{1}$ is corrugation depth.

We assume that the system parameters satisfy to the Bragg resonance condition

$$
\bar{h}_{z}=\bar{h}_{x}=\bar{h},
$$

Fig. 1. Scheme of oversized SWO with $2 \mathrm{D}$ corrugated structure

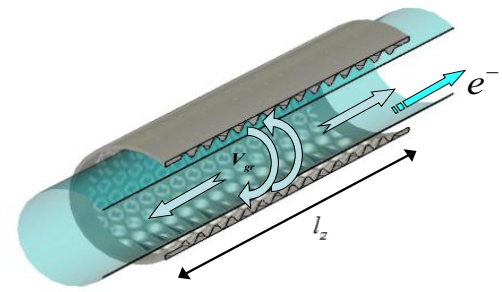

where $\bar{h}_{x}=\bar{M} / r_{0}$. If the curvature of the waveguide is small, i.e. its radius $r_{0}$ is substantially larger than the wavelength $\lambda$, one is able to introduce new coordinate $x=r_{0} \varphi$ and to use a quasi-planar model. On the 2D corrugated surface (1) under resonance condition (2), coupling and mutual scattering of the four partial quasi-optical wave-beams occurs

$$
\vec{H}=\operatorname{Re}\left[\left(\vec{x}_{0}\left(C_{z}^{+} e^{-i \bar{h} z}+C_{z}^{-} e^{i \bar{h} z}\right)+\vec{z}_{0}\left(C_{z}^{+} e^{-i \bar{h} x}+C_{z}^{-} e^{i \bar{h} x}\right) e^{i \bar{\omega} t}\right] .\right.
$$

Two of these beams $C_{z}^{ \pm}$propagate along the longitudinal $\pm z$ directions and the other two $C_{x}^{ \pm}$propagate along the transverse (azimuthal) $\pm x( \pm \varphi)$ directions. Radial coordinate $y$ is directed normally to the surface of the conductor. Choosing Bragg frequency $\bar{\omega}=\bar{h} c$ as a reference frequency, the excitation of surface waves by the electron beam can be described by the following system of equations [5]

$$
\begin{gathered}
i \frac{\partial^{2} \hat{C}_{z}^{+}}{\partial Y^{2}}+\frac{\partial \hat{C}_{z}^{+}}{\partial Z}+\frac{\partial \hat{C}_{z}^{+}}{\partial \tau}=i \hat{\alpha}\left(\hat{C}_{x}^{+}+\hat{C}_{x}^{-}\right) \delta(Y)-\frac{1}{B_{e}} \frac{\partial}{\partial Y} J F(Y), \\
i \frac{\partial^{2} \hat{C}_{z}^{-}}{\partial Y^{2}}-\frac{\partial \hat{C}_{z}^{-}}{\partial Z}+\frac{\partial \hat{C}_{z}^{-}}{\partial \tau}=i \hat{\alpha}\left(\hat{C}_{x}^{+}+\hat{C}_{x}^{-}\right) \delta(Y) \\
i \frac{\partial^{2} \hat{C}_{x}^{ \pm}}{\partial Y^{2}} \pm \frac{\partial \hat{C}_{x}^{ \pm}}{\partial X}+\frac{\partial \hat{C}_{x}^{ \pm}}{\partial \tau}=i \hat{\alpha}\left(\hat{C}_{z}^{+}+\hat{C}_{z}^{-}\right) \delta(Y)
\end{gathered}
$$

where $\delta(Y)$ is the delta function, $F(Y)$ is a function defining the radial current density of the electron beam with an effective thickness of $B_{e}$. Amplitude of the RF current

$$
J=\frac{1}{\pi} \int_{0}^{2 \pi} e^{-i \theta} \mathrm{d} \theta_{0}
$$

can be found from the electron motion equations

$$
\left(\frac{\partial}{\partial Z}+\frac{1}{\beta_{0}} \frac{\partial}{\partial \tau}\right)^{2} \theta=\operatorname{Re}\left[\frac{\partial \hat{C}_{z}^{+}}{\partial Y} e^{i \theta}\right] .
$$

Here $\theta$ is the electron phase with respect to the forward partial wave, $\Delta$ is the synchronism detuning on the carrier frequency $(\Delta>0)$, which may vary with changes in the particle energy. Synchronous interaction with a rectilinear electron beam arises due to the formation of an evanescent slow wave. In (4), (5) we used the following normalized variables and parameters: $Z=G \bar{h} z$, $X=G \bar{h} x, Y=\sqrt{2 G} \bar{h} y, \tau=G \bar{\omega} t, G$ is the gain parame- 
ter, $\hat{\alpha}$ is normalized wave coupling parameter at the corrugation (1). Due to the cylindrical geometry of the cavity the wave-beam amplitudes should satisfy the cyclic boundary conditions

$$
\hat{C}_{x, z}^{ \pm}\left(X+L_{x} ; Y ; Z\right)=\hat{C}_{x, z}^{ \pm}(X ; Y ; Z),
$$

where $L_{x}=2 \pi \bar{h} G r_{0}$ is perimeter of the structure. These conditions allow solution of Eqs. (4) to be expanded in a Fourier series

$$
\hat{C}_{x, z}^{ \pm}(X ; Y ; Z ; \tau)=\sum_{m=-\infty}^{+\infty} \hat{C}_{x, z}^{ \pm, m}(Y ; Z ; \tau) e^{2 \pi i m X / L_{x}},
$$

considering each harmonic as a mode with an azimuthal index $m$. Boundary conditions for Eqs.(4) at the edges of the corrugated region correspond to the absence of external energy fluxes.

We used Eqs.(4)-(7) to simulate operation of powerful relativistic surface-wave oscillator at the wavelength of $8 \mathrm{~mm}$ driven by relativistic electron beam of $1 \mathrm{MeV}$, linear current density of $100 \mathrm{~A} / \mathrm{cm}$ and beam radius $r_{0}=2 \mathrm{~cm}$. We consider the 2D corrugation of the period $d_{\mathrm{z}}=7 \mathrm{~mm}$ and depth $r_{1}=1 \mathrm{~mm}$ with the length of corrugated section $l_{\mathrm{z}}=21 \mathrm{~cm}$. These parameters correspond to the normalized values $G \approx 0.01, \hat{\alpha}=2.2, \Delta \approx 8, L_{\mathrm{z}}=1.5, L_{\mathrm{x}}=1$.

Figure 2 shows results of simulations of nonlinear stage of interaction. The temporal dependencies of amplitudes of different azimuthal components of the wavebeam $\hat{C}_{z}^{-}$at various times of accelerating voltage front are presented. With zero rise time of the front we see excitation of a single symmetric mode (Fig. 2a). Amplitudes of others non-symmetric modes are negligibly small. However with the front time of 200 ns (Fig. 2b) the azimuthally non-symmetric mode suppresses symmetric mode and sets on.
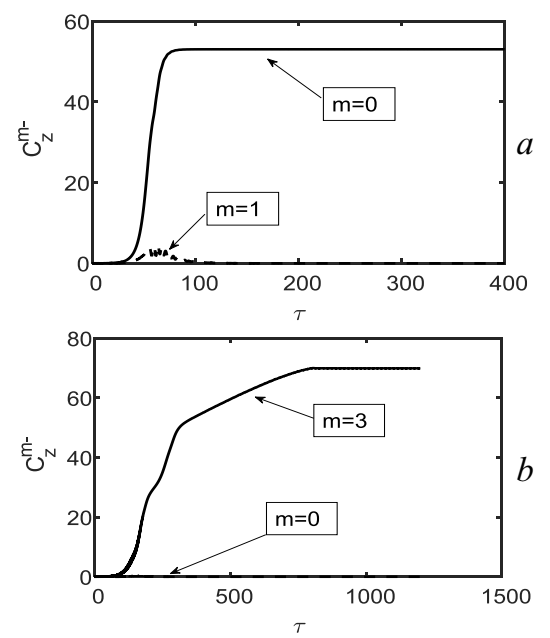

Fig. 2. Temporal dependencies of amplitudes of different azimuthal components of the wave-beam $\hat{C}_{z}^{-}$at duration of the accelerating voltage front of (a) 0 and (b) $200 \mathrm{~ns}$

\section{Experimental results}

Experiments with Ka-band 2D-SWO were carried out based on microsecond accelerator "Saturn" (IAP RAS). Tubular electron beam $100 \mathrm{~A} / 200-300 \mathrm{keV}$ was formed from a thermionic cathode with a diameter of $5 \mathrm{~cm}$. As a result of the experiments, stable narrow-band generation in strongly oversized system (perimeter-towavelength ratio is about 16) using periodical structure with $2 \mathrm{D}$ corrugation has been observed in the design region of parameters (Fig. 3). Calorimetric measurement indicates the power level of $1.5-2 \mathrm{MW}$ in up to $200 \mathrm{~ns}$ pulses, that corresponds to electron efficiency of $\sim 5 \%$. High stability of the oscillation frequency with respect to variation of the electron beam parameters was demonstrated. In accordance with the results of simulations, frequency stabilization is caused by the presence of azimuthal energy fluxes provided by 2D corrugated structure.

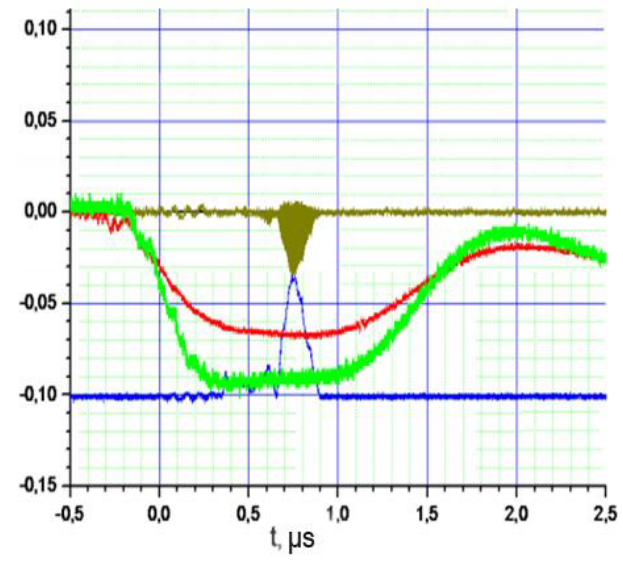

$a$

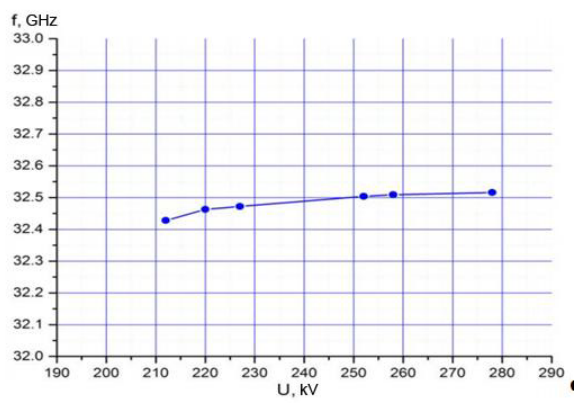

$b$

Fig. 3. (a) Typical oscilloscope traces of the accelerating voltage (green curve), beam current (red), heterodyne signal (brown) and output RF pulse (blue). (b) Dependence of the radiation frequency on the accelerating voltage

Acknowledgments. This work is partially supported by the Russian Foundation for Basic Research (grant \#1602-00890).

\section{References}

1. N.S. Ginzburg, N.Yu. Peskov, A.S. Sergeev Dynamics of free-electron lasers with two-dimensional distributed feedback // Optics Commun. 1994. V.112. P.151.

2. I.V. Konoplev, A.W. Cross, A.D.R. Phelps, e.a. Co-axial Free-Electron Maser based on two-dimensional distributed feedback. // Phys. Rev. E. 2007. V.76. P.056406.

3. A.V. Arzhannikov, N.S. Ginzburg, P.V. Kalinin, e.a. Using two-dimensional distributed feedback for synchronization of radiation from two parallel-sheet electron beams in FEM // Phys. Rev. Lett. 2016. V.117. P.114801.

4. N.S. Ginzburg, E.V. Ilyakov, I.S. Kulagin, e.a. Synchronization of radiation in an oversized coaxial Ka-band BWO using two-dimensional Bragg structure // Phys. Rev. Accel. Beams. 2015. V.18. P.120701.

5. N.S. Ginzburg, A.M. Malkin, A.S. Sergeev, V.Yu. Zaslavsky Oversized co-axial and cylindrical surface-wave oscillators with 2D periodical grating (quasi-optical model) // J. Appl. Phys. 2013. V.113. P.104504. 